\title{
Bases para el estudio de la dinámica discursiva en la comunidad jurídica chilena*
}

\author{
María Cecilia García Petit** \\ Claudio Agüero San Juan***
}

\begin{abstract}
RESUMEN
Este artículo reflexiona sobre cómo la comunidad jurídica chilena se relaciona con la sociedad mediante la producción de discursos. El objetivo es fijar las bases de un modelo de las relaciones discursivas entre los expertos en derecho y los legos. Para lograr este objetivo se analiza cómo los miembros de la comunidad jurídica crean, fijan y transmiten los conocimientos jurídicos usando, principalmente, textos que responden a géneros discursivos. Se concluye que el análisis de la comunidad jurídica debe ser sensible a la dinámica discursiva de la comunidad y a cómo ella intercambia bienes y servicios por medio del uso de textos.
\end{abstract}

Dinámica discursiva - géneros especializados - discurso jurídico comunidad jurídica chilena

\section{Bases for the study of discursive dynamics on chilean legal community}

\begin{abstract}
This article reflects on how the Chilean legal community relates to the society through the production of discourse. The aim is to establish the basis of a model of discourse relations between legal experts and laymen. To achieve this goal we analyze how members of the legal community create, set and using legal knowledge transmitted mainly responding to texts discourse genres. We conclude that the analysis of the legal community should be sensitive to the discursive dynamics of the community and how they exchange goods and services through the use of texts.
\end{abstract}

Discursive dynamic - specialized genres - legal discourse - chilean legal community

* Investigación realizada en el marco del proyecto FONDECYT regular No 1110332.

** Abogada y estudiante del Programa de Doctorado en Ciencias Humanas c/m en discurso y Cultura, Universidad Austral de Chile. Campus Isla Teja, Valdivia, Chile. Correo electrónico: ceciliagarciapetit@gmail.com

*** Abogado, Máster en Derecho y Doctor en Ciencias Humanas. Profesor de las facultades de derecho de las universidades Alberto Hurtado, Diego Portales y de Valparaíso. Cienfuegos 41, Santiago Centro, Chile. Correo electrónico: aguero.claudio@gmail.com

Artículo recibido el 13 de noviembre de 2013 y aceptado para su publicación por el Comité Editorial el 28 de mayo de 2014. 


\section{INTRODUCCIÓN $^{1}$}

$\mathrm{E}$ n Chile no contamos con investigaciones sistemáticas sobre la comunidad de los abogados ni sobre las relaciones entre estos profesionales y la sociedad. Desconocemos, por ejemplo, cómo se organiza la enseñanza del derecho; cómo se socializa profesionalmente a los miembros nóveles de la comunidad jurídica; cómo se distribuyen los incentivos sociales entre los abogados; cómo los abogados responden a las expectativas de los ciudadanos y de las instituciones políticas y cómo fijan, transmiten y reproducen los conocimientos quienes son expertos en derecho. El amplio margen de desconocimiento es problemático por varias razones. La menos controvertible ya la conocía Tocqueville: los abogados son importantes para el funcionamiento de las instituciones sociales y para el logro de los ideales políticos del Estado de Derecho y de la Democracia ${ }^{2}$.

Este artículo busca reducir el margen de desconocimiento sobre la comunidad jurídica chilena por medio de un modelo elemental para el estudio de su dinámica discursiva. El modelo propuesto combina dos criterios: uno etnográfico que distingue a los abogados como un grupo de expertos en derecho y uno discursivo que diferencia los textos jurídicos como aquellos que son identitarios de la profesión porque los abogados los elaboran y/o consumen.

El modelo propuesto es el primer paso de una investigación de largo aliento que pretende desarrollar un estudio empírico sobre la comunidad jurídica chilena. Entonces, debe ser entendido como una construcción epistemológica a priori; el paso previo al diseño y aplicación de instrumentos de recopilación de información como entrevistas, encuestas, focus group, construcción de bases de datos cuantitativas y análisis de textos dogmáticos o jurisprudenciales. El modelo que se presenta no es el producto de un trabajo de campo, sino que se trata de un modelo mínimo, flexible, émico (emic) y cualitativo. Es mínimo porque solo busca fijar las relaciones conceptuales indispensables para enfrentar el diseño de instrumentos de investigación. Es flexible porque aunque es pensado para la investigación de los abogados chilenos podría ser usado (con ciertos ajustes) para estudiar a otras comunidades profesionales nacionales o extranjeras. Es émico porque sus autores son abogados y, entonces, está diseñado desde el interior de la comunidad jurídica ${ }^{3}, \mathrm{y}$

\footnotetext{
${ }^{1}$ Los autores agradecen los comentarios de Daniel Palacios, Jorge Larroucau, Rodrigo Coloma, Flavia Carbonell, Federico Arena, Michel Duquesnoy, María Beatriz Arriagada, Cristián Santibáñez, Hugo Herrera, José Julio León y Juan Pablo Zambrano. También agradecen las observaciones de otros asistentes a los seminarios de investigación realizados en las universidades Alberto Hurtado y Diego Portales donde se discutieron borradores de este trabajo. Advertimos que no nos hacemos cargo de muchas de las observaciones formuladas en esas sesiones solo por razones de espacio editorial. Los errores que persisten en el texto no les son reprochables a los comentaristas.

2 Tocqueville, A. La democracia en América. Fondo de Cultura Económica, México DF., 2006.

${ }^{3}$ La distinción entre émico (emic) y ético (etic) fue usada por primera vez por Pike. Luego fue adoptada por Harris y, desde mediados de la década de los setenta del siglo pasado, adquirió carta de ciudadanía en los estudios sociales. En términos generales, una descripción emic de una práctica social es aquella que es significativa para quienes participan de esa práctica, mientras que una descripción etic es la que es juzgada
} 
es cualitativo porque su orientación busca posibilitar la investigación social cruzando los datos entregados por las personas con el análisis de los textos que la comunidad usa.

La perspectiva etnográfica que es privilegiada en este modelo es complementada con un enfoque de análisis del discurso ${ }^{4}$. Este enfoque complementario se justifica en la indisoluble relación entre las normas y el lenguaje. Si más allá del lenguaje no es posible pensar en las normas, es indispensable describir cómo ese lenguaje, instanciado en textos, comunica también otros propósitos comunicativos. Habida consideración que las funciones del lenguaje no se disocian unas de otras, todos los textos jurídicos como las sentencias judiciales, los contratos, los testamentos y las leyes combinan varios propósitos, porque estos textos no solo sirven para fijar relaciones jurídicas, sino que también nos informan sobre una forma de entender la vida social y sobre qué es considerado valioso por un grupo humano en un tiempo determinado 5 .

La combinación de propósitos comunicativos que se instancia en un texto no es arbitraria ni azarosa ni está completamente determinada por exigencias internas al texto. La combinación y su intensidad está determinada por las necesidades socioculturales que el texto busca satisfacer, lo que releva la importancia de completar los puntos de vista etnográfico y discursivo para determinar cómo el texto se usa por el grupo humano que lo ha creado ${ }^{6}$. Así, para entender qué función(es) cumple(n) la(s) parte(s) de un texto es necesario atender a cómo ella(s) interactúa(n) y cómo el texto es usado en la dinámica social. Esto significa que para comprender cómo y por qué se organiza un texto de un modo determinado es necesario saber cómo él se inserta en la sociedad.

De acuerdo con estas ideas, la importancia del trabajo que se presenta no solo radica en hacer viable el concepto de cultura jurídica en el sentido que Nelken ${ }^{7}$ da a esa expresión. Además, el modelo propuesto permite enfocar cómo las variables políticas, sociales y culturales modulan la implementación de los ideales asociados al sistema jurídico ${ }^{8}$. Sin perjuicio del sinnúmero de matices que existen entre el modelo propuesto y otros

como significativa por quienes no participan de la práctica social descrita. Esto implica que las descripciones emic no pretenden ser neutrales culturalmente, en tanto que las etic tienen tal pretensión. Pike, K. (ed.). Language in Relation to a Unified Theory of Structure of Human Bebavior, Mouton, The Hague, Netherlands, 1967; Harris, M. "History and Significance of the Emic/Etic Distinction", en Annual Review of Anthropology, 5, 1976, pp. 329-350; Harris, M. “Chapter Two: The Epistemology of Cultural Materialism”, en Cultural Materialism: The Struggle for a Science of Culture. Random House, New York, 1980, pp. 29-45; Headland, T.; Pike, K.; Harris, M. (eds). Emics and Etics: The Insider/Outsider Debate, Sage Publications, Beverly Hills, California, 1990; Kitayama, S. \& Cohen, D. Handbook of Cultural Psychology, Guilford Press, New York, 2007. ${ }^{4}$ Gibbons, J. Language and the Law. Longman, Londres, 1994; Fletcher, G. "Law", en Smith, B. (ed.) John Searle. Cambridge University Press, Cambridge, 2003; Galdia, M. Legal Linguistics. Peter Lang, Frankfurt, 2009.

${ }^{5}$ Gee, J. An Introduction to Discourse Analysis: Theory and Method. Routledge, Londres, 1999.

${ }^{6}$ Wodak, R \& Meyer, M (Eds). Methods of Critical Discourse Analysis. Sage Publications, Londres, 2001.

${ }^{7}$ Nelken, D. "Using the concept of legal culture", en Australian Journal of Legal Philosphy, No 29, pp. 1-28. Disponible en: http://www.law.berkeley.edu/files/Nelken_-_Using_the_Concept_of_Legal_Culture.pdf [consultado el 11 de noviembre de 2013].

${ }^{8}$ Klein, G. Sources of power: How people make decisions. MIT Press, Boston, 1994; Travers, M. \& Manzo, J. E. Law in action: Ethnomethodological and conversation analytical approaches to law. Ashgate, Aldershot, 1997; Conley, J.M. \& O'Barr, W.M. Rules versus relationships. The ethnography of legal discourse. Chicago: The University 
estudios empíricos que circulan en el Common Law merece la pena destacar dos de ellos: a) la tesis que afirma que es importante analizar la estructura de los textos jurídicos y su relación con otros textos (jurídicos y no jurídicos) y, b) la tesis que sostiene que los textos jurídicos expresan relaciones sociales subyacentes. La primera tesis marca una diferencia con los estudios ideológicos y/o argumentales que configuran las categorías de análisis sin considerar la morfología y el funcionamiento del texto analizado y sus relaciones con otros textos adyacentes. La segunda tesis permite distanciar al modelo propuesto de aquellos análisis que dejan de lado las variables sociales porque suponen que las actitudes ideológicas ${ }^{9}$ de los abogados están distribuidas igualitariamente, o porque se concentran solo en el comportamiento de un grupo de abogados (por ejemplo, los jueces del Tribunal Constitucional o los jueces de las Cortes de Apelaciones) y no buscan desvelar los lazos (textuales y sociales) que ligan a los miembros del grupo con otros tipos de abogados o grupos sociales.

\section{SOBRE EL CONCEPTO DE MODELO}

En este trabajo el concepto de modelo es usado en un sentido específico. Siguiendo a Bailer-Jones ${ }^{10}$ entendemos por modelo una descripción interpretativa simplificada de las relaciones discursivas entre los miembros de la comunidad jurídica (que son expertos en derecho) y entre ellos y los miembros de la comunidad normativa que son legos o semilegos en asuntos jurídicos.

El modelo fue pensado con propósitos etnográficos moderados, es decir, el modelo ayuda a describir cómo, dentro de una sociedad como la chilena, los abogados se convierten en una comunidad de profesionales que crea y usa textos especializados (académicos y profesionales); cómo esos textos circulan entre los integrantes del grupo social y cómo la circulación de los textos es reflejo de las relaciones sociales entre los miembros de la comunidad. La pretensión de un modelo como el que se propone aquí no es describir toda la realidad ni representar toda la complejidad de la vida social, sino que simplificar la interacción entre los abogados y entre ellos y la ciudadanía.

La simplicidad exige construir definiciones rígidas que muchas veces son reconstrucciones de uno o varios significados difusos (fuzzy). En un plano metodológico el modelo que este trabajo propone es a priori, es decir, se concentra en delinear una red de significados teóricos y no ha sido construido considerando datos empíricos en profundidad. En este sentido, el modelo propuesto es provisional y falseable. Hay varias razones para optar por un modelo teórico de este tipo, pero enunciaremos solo dos de

of Chicago Press, 1998; Nader, L. The life of the law. Anthropological projects. University of California Press, Berkeley, 2002.

9 Usamos la expresión actitud ideológica en sentido amplio como un conjunto más o menos coherente de creencias sobre qué es correcto en términos morales, políticos, religiosos y, en general, sociales.

${ }^{10}$ Bailer-Jones, D. "Models, Metaphors and Analogies", en Machamer, P. \& Silberstein, M. (eds.), The Blackwell Guide to the Philosophy of Science. Oxford, Blackwell Publishers, 2002, pp. 108-127. 
ellas. La primera se vincula a la ausencia de información etnográfica fiable sobre la integración y funcionamiento de la comunidad jurídica chilena, lo que exige tomar decisiones apriorísticas antes de iniciar cualquier tipo de exploración empírica ${ }^{11}$. La segunda razón dice relación con el desarraigo de este tipo de investigaciones en Chile. En pocas palabras: no hay investigaciones sobre cómo se elaboran y circulan los textos jurídicos en nuestro país.

La pretensión descriptiva del modelo no significa afirmar su imparcialidad. En investigaciones posteriores, se aspira a lograr una etnografía moderada. El modelo solo busca cumplir con el estándar de arbitrariedad crítica flexible (flexible critical arbitrariness) propuesto por Barker y Galasinski ${ }^{12}$, es decir, asegurar que el lector está consciente de la posición desde donde se analizan los textos jurídicos. La propuesta tampoco elude el problema de la ideología en el discurso. En este artículo el término ideología es entendido como un conjunto de representaciones sociales compartidas por los miembros de un grupo y que son usadas por ellos para realizar las prácticas sociales diarias. Es decir, todos los usos del lenguaje son ideológicos, porque las ideologías son los supuestos de sentido común que forman perspectivas capaces de limar las contradicciones, dilemas y antagonismos de las prácticas sociales, de manera que estén en concordancia con los intereses y proyectos del grupo dominante ${ }^{13}$.

Tratándose de un modelo sobre la relación entre la comunidad jurídica y la sociedad, la primera sección del trabajo formula un discurso que no es susceptible de verdad ni falsedad, aunque pretende contener algunas proposiciones verdaderas sobre las relaciones en cuestión. Por otro lado, es importante advertir que la función del modelo no es describir todas las posibles implicancias teóricas y prácticas de las relaciones que se establecen entre los abogados y los ciudadanos. Su única pretensión es mostrar cómo se producen los textos jurídicos orales y escritos y cómo son utilizados dentro de la sociedad chilena por los abogados para intercambiar bienes y servicios.

\footnotetext{
${ }^{11}$ La única investigación sobre los abogados chilenos es la realizada por De la Maza, pero no se trata de una investigación empírica. En relación con el uso de los textos jurídicos, la única investigación es el trabajo de Matus sobre la comunidad de expertos en derecho penal. De la Maza, I. Los abogados chilenos: entre el Estado y el mercado. Juris Science master's thesis, Programa for International Legal Studies, Stanford University, publicada bajo el mismo título en el número 10 de la Colección de Informes de Investigación de la Universidad Diego Portales, enero de 2002. Disponible en el sitio web: http://www.udp.cl/descargas/facultades_carreras/derecho/pdf/ investigaciones/Informes_derecho_Informes_de_investigacion/10_Losabogados_Estadomercado_Inig_delaMaza. pdf [consultado el 30 de octubre de 2013].

Matus, J. "El positivismo en el derecho penal chileno. Análisis sincrónico y diacrónico de una doctrina de principios del siglo XX que se mantiene vigente”, en Revista de Derecho, vol. XX N 1, 2007, pp. 175-203. Disponible en: http://www.scielo.cl/pdf/revider/v20n1/art08.pdf [consultado el 30 de octubre de 2003] Matus, J. y Carnevalli, R. "Análisis descriptivo y cuantitativo de los artículos de Derecho penal y Criminología de autores chilenos en Revistas publicadas en Chile (1885-2006)”, en Revista Política criminal, Vol. 2, No 3 , julio, 2007, D2, pp. 1-138.

12 Barker, C. y Galasinski, D. Cultural Studies and Discourse Analysis. A Dialogue on Language and Identity, Sage Publications, Londres, 2001, p. 64.

${ }^{13}$ Chouliaraki, L. \& Fairclough, N. Discourse in late modernity. Retbinking critical discourse analysis. Edinburgh University Press, Edimburgo, 1999.
} 
El modelo debiese permitir explorar cuáles son los destinatarios preferentes de un texto jurídico y cómo la elección de ese destinatario dificulta o facilita la accesibilidad del mensaje que ella busca transmitir a otros grupos. El modelo no se ocupa de formular criterios sobre cómo usar bien el derecho, sino de cómo describir la tensión social que existe entre, por un lado, el requerimiento de los abogados por un lenguaje preciso, técnico y económico y, por otro, la demanda social de los ciudadanos por un lenguaje simple, llano y accesible.

\section{LA SOCIEDAD Y SU LENGUAJE}

En los grupos humanos que llamamos sociedades, todas las prácticas de sus miembros replican los saberes que permiten al grupo interpretar normativamente (comprender) las acciones intencionales de los individuos. En principio, las acciones puramente mentales como pensar, imaginar o soñar carecen de relevancia normativa para las normas sociales y jurídicas porque no poseen una dimensión material que las concretice. Asimismo, las acciones que no tienen una dimensión motivacional como los estornudos, los comportamientos reflejos y los tics nerviosos tampoco importan demasiado.

Para conocer el comportamiento normativo de una sociedad es central saber cómo son interpretadas las acciones que Von Wright llamaba intencionales ${ }^{14}$, es decir, aquellas que realiza un agente buscando materializar un propósito, intención o fin. Son acciones intencionales las tareas educacionales, religiosas, políticas, científicas y jurídicas, entre otras prácticas sociales. Todas ellas solo pueden ser entendidas si conocemos -usando la expresión de Von Wright- la comunidad de vida que les dio origen ${ }^{15}$. Esto significa que la única forma de conocer la intención que motiva estas acciones es comprenderlas (understanding).

La idea de comprensión cuestiona la posibilidad de fragmentar la conducta intencional en actos mentales y movimientos corporales vinculados por reglas asociativas ${ }^{16}$. Esto significa que para establecer que un agente tuvo una determinada intención al realizar una acción es necesario construir analogías con otras acciones semejantes que han tenido resultados similares. Además, es necesario considerar que en todas estas acciones el lenguaje funciona como una herramienta con la que se constituye la sociedad y que, al mismo tiempo, permite a sus miembros insertarse socialmente ${ }^{17}$.

El lenguaje es una institución política y dominarlo exige tener conciencia explícita de qué recursos lingüísticos están socialmente asociados a diferentes contextos de

\footnotetext{
${ }^{14}$ Von Wright, G. Explicación y comprensión. Alianza, Madrid, 1987.

${ }^{15}$ Von Wright, G. Explicación y comprensión. Alianza, Madrid, 1987. Von Wright, G. Ensayos sobre explicación y comprensión. Alianza, Madrid, 1980.

${ }^{16}$ Von Wright, G. Explicación y comprensión. Alianza, Madrid, 1987.

${ }^{17}$ Oteíza, T. El discurso pedagógico de la historia: un análisis lingüístico sobre la construcción ideológica de la historia de Chile (1970-2001), Frasis Editores, Santiago, 2006, p. 53.
} 
interacción y campos de conocimiento. Lemke ${ }^{18}$, igual que Von Wright ${ }^{19}$, postula que todos los significados son realizados al interior de una comunidad y que es imposible analizar los significados disociados de los significantes, ya que el significado y significante surgen sincrónicamente. Esto implica que todas las prácticas sociales crean y reproducen significados dentro de una comunidad y que no es posible separar las dimensiones social, histórica, normativa y política del lenguaje, porque todo cambio de las prácticas lingüísticas es un cambio social y viceversa.

Halliday y Martin van más allá que Von Wright ${ }^{20}$. Ellos han afirmado que la cultura y el lenguaje coevolucionan en la misma relación en la que dentro del lenguaje, significado y expresión coevolucionan. Aunque los miembros de una comunidad construyen realidades sociales usando deliberadamente el lenguaje, los textos producidos no son la manifestación de acciones intencionales individuales y aisladas, sino que son instancias de expresión de una red comunitaria de significados que el agente conoce y usa con un propósito sociocultural. Así, los cambios del lenguaje en las prácticas sociales pueden ser entendidos como una actividad de creación, fijación y transmisión de significados con el lenguaje, y la red de significados (el conjunto de relaciones intertextuales) es un espejo de la red social que sustenta al grupo humano y de los intercambios entre sus participantes. La red de significado es entonces un soporte que sustenta la cultura y, al mismo tiempo, es el medio de expresión de la cultura porque todas las prácticas de los participantes se vinculan con otras prácticas precedentes, simultáneas y futuras.

Estudiar así la cultura permite entender por qué las prácticas sociales tienen una carga normativa flexible, dinámica y dependiente de otras prácticas a las que están culturalmente atadas. Naturalmente, todo este fenómeno es parcialmente invisible para los participantes e incomprensible para quienes no son miembros de la comunidad. Por ello, Hassan nos advierte que mientras menos visible sea el papel del lenguaje en el desarrollo de la conciencia, y mientras menos clara sea la relación de este en cambiar las sociedades, más fácil es mantener el statu quo ${ }^{21}$.

\section{LA COMUNIDADES NORMATIVA Y JURÍDICA}

El modelo considera que todos los miembros de un grupo social que son capaces de realizar acciones intencionales e interpretar las acciones de otros pertenecen a una misma

\footnotetext{
${ }^{18}$ Lemke, J.L. Using language in the classroom. Oxford University Press, Oxford, 1989.

${ }^{19}$ Von Wright, G. Explicación y comprensión. Alianza, Madrid, 1987.

${ }^{20}$ Halliday, M. \& Martin, J. Writing science. Literacy and discursive power. University of Pittsburgh Press, Pittsburgh, 1993, p. 11.

${ }^{21}$ Hassan, R. "Society, language and the mind: the meta-dialogism of Basil Bernsteins theory", en Christie, F. (ed). Pedagogy and the shaping of consciousness. Linguistic and social processes. Continuum, Londres, 1999 , p. 30.
} 
comunidad normativa ${ }^{22}$. Esta noción es propuesta para sustituir el concepto de sociedad con dos objetivos: i) enfatizar que lo relevante para caracterizar a los integrantes de un grupo es su capacidad de actuar intencionalmente y para atribuir un significado a las acciones de otros y, ii) evitar el isomorfismo entre el modelo y el fenómeno ${ }^{23}$.

\subsection{El concepto de comunidad normativa}

Todos los miembros de una comunidad normativa son competentes para realizar, por ejemplo, algunas de las siguientes evaluaciones (aunque no puedan explicitarlas convenientemente): i) determinar cuándo se ha realizado una acción, ii) interpretar correctamente cuándo la conducta de uno de los participantes ha sido (o debería haber sido) censurada por haber roto alguna regla social, iii) saber cuándo una conducta ha sido realizada según las normas que gobiernan la comunidad y, iv) saber cuándo la intención tras una acción se opone a los valores sociales dominantes, entre otras evaluaciones. Esta competencia evaluativa permite observar que todos quienes comparten un sistema semiótico que les permite interpretar un contenido con carga normativa (como por ejemplo la intención de la acción) a partir de ciertos signos (como el resultado de una acción) son miembros de una misma comunidad normativa ${ }^{24}$.

Mientras que el observador externo solo puede observar coincidencias evaluativas, como la existencia de reglas de cortesía y de trato social o de acuerdos flexibles sobre la moralidad o religiosidad de algunos actos, entre otras prácticas sociales, el participante comprende cuál es el sentido de esas coincidencias, porque está atado por un lazo (a veces invisible para él mismo) que le permite saber por qué, cuándo, cómo y en qué los otros miembros del grupo están de acuerdo. En este sentido, los miembros de una comunidad normativa empatizan unos con otros, porque el sustrato cultural compartido les permite representarse las creencias, las opiniones, las decisiones y las posibilidades de acción de los demás.

La competencia para interpretar normativamente ciertas acciones y para empatizar con los otros define la membresía para integrar una comunidad normativa ${ }^{25}$. Como es posible aprender estas destrezas, es posible que un individuo sea capaz de dominar el sistema semiótico de dos o más comunidades normativas. Ahora bien, dentro de cada comunidad normativa se pueden distinguir subconjuntos más o menos difusos. Los criterios para definir estos subconjuntos pueden ser tan variados como los objetivos de

\footnotetext{
${ }^{22}$ Una aplicación posible del modelo de comunidad normativa está en los estudios de Folk Psychology y de Filosofía Experimental.

${ }^{23}$ Hugo Herrera y Cristián Santibáñez nos han formulado interesantes observaciones en este punto. En particular sobre el rol agencial de la membresía en la comunidad normativa y sobre la filosofía del lenguaje subyacente al modelo. Ambas observaciones no han sido asumidas en este trabajo pues requieren de un desarrollo conceptual in extenso.

${ }^{24}$ Lotman, Y. Estructura del texto artístico. ITSMO, Madrid, 1982.

${ }^{25}$ Federico Arena ha resaltado que este criterio de membresía le parece débil. Estamos de acuerdo con la observación, pero pensamos que el trabajo de campo debería suministrar información adicional para fortalecer el criterio.
} 
investigación a los que ellos sirven. En el modelo que proponemos el criterio de agrupación es la profesionalización de la actividad jurídica.

\subsection{La comunidad jurídica}

Llamamos comunidad jurídica al conjunto de personas que, integrando una o más comunidades normativas, son reconocidos por otros miembros de esas comunidades como expertos en derecho. En sociedades como la chilena, son miembros de la comunidad jurídica aquellos individuos que estudiaron derecho a nivel universitario y que lo practican profesionalmente como abogados en diversos sectores laborales o productivos dentro de una o más comunidades normativas.

La elección del criterio de composición de la comunidad jurídica no debe ser comprendido como un estándar cualitativo, es decir, no implica sostener la tesis que afirma que son los abogados los individuos mejor calificados socialmente para hacer las mejores evaluaciones de la carga normativa de una acción dentro de una comunidad normativa. El criterio es solo formal y, en comunidades como la chilena, se cumple con tres requisitos copulativos: i) tener formación académica en derecho a nivel universitario, ii) poseer el título de abogado y, iii) ejercer profesionalmente como abogado. Así, la comunidad jurídica se define operacionalmente como un grupo de expertos, porque sus miembros son los profesionales del derecho que practican la disciplina de algún modo.

\subsection{La organización de la comunidad jurídica}

Modelar la comunidad jurídica exige reflexionar sobre la organización del trabajo y la distribución de la especialización dentro de ella. Siguiendo el punto de vista etnográfico de los trabajos de Adler y de Adler y Gil, el mercado de trabajo de los abogados puede dividirse en cuatro grupos: i) el sector público, ii) la elite profesional, iii) el sector privado y iv) la academia ${ }^{26}$.

El sector público agrupa a todos los abogados que integran los niveles bajos y medios de los servicios públicos y la burocracia estatal. Estos funcionarios pueden ocupar posiciones con poder de decisión pero que no pertenecen a la elite técnica o política de los expertos en derecho. Su principal tarea es ocuparse de problemas técnicos para sostener el sistema jurídico en todos los organismos del Estado. Ejemplos de este tipo de abogado son: jueces, defensores penales, fiscales, abogados de municipalidades, abogados en Secretarías Ministeriales, etcétera.

La elite profesional es un grupo de abogados que por diversas razones es privilegiado y exclusivo y que tiene el poder de influir en los otros miembros de la comunidad jurídica. La pertenencia a este subgrupo de abogados puede estar determinada por algunas de las siguientes vías: obtención de cargos de votación popular; ejercer cargos

\footnotetext{
${ }^{26}$ Adler, L. Lo formal y lo informal en las sociedades contemporáneas. Centro de Investigaciones Barros Arana, Santiago, 2008; Adler, L. \& Gil-Mendieta, J. "El neoliberalismo y los cambios en la elite de poder en México", en Redes: Revista hispana para el análisis de redes sociales, 1, 2002, pp. 1-23.
} 
de poder en el sistema jurídico como los ministros de la Corte Suprema o en las Cortes de Apelaciones; ocupar puestos como jefe de servicio en la Administración Pública y en órganos descentralizados funcionalmente; por poseer prestigio social al interior de la comunidad profesional; por poseer conocimientos especializados o tecnocráticos de alto nivel como, por ejemplo, tener formación a nivel de postgrado en las mejores universidades; por contar con redes horizontales (son hijos, parientes o amigos íntimos de un miembro de la elite) o por tener redes verticales (gestionan lealtades con abogados de menor prestigio o jerarquía ${ }^{27}$.

El sector privado se compone de todos aquellos abogados que ejercen de forma liberal en estudios, firmas o despachos de abogados, ya sea que se especialicen en litigios o en asesorías corporativas.

Finalmente, los abogados que se dedican a la academia son aquellos que desarrollan docencia e investigación en la universidad y en otros organismos de investigación.

Aunque los cuatro grupos de abogados comparten cierto conjunto de conocimientos sobre las normas que regulan la vida social dentro de la comunidad normativa y la vida profesional en la comunidad jurídica, esto no significa que compartan todo un conjunto homogéneo de convenciones para interactuar discursivamente; que conozcan los criterios de membresía para ser admitidos como miembros de cada grupo o que tengan claro qué tipo de bienes son valiosos para cada grupo.

Creemos que la profundidad etnográfica de los trabajos de Adler y de Adler y Gil entorpece el estudio de la circulación de los textos entre los diferentes tipos de abogados y entre ellos y los legos. Este objetivo exige sacrificar la descripción etnográfica en favor de la simplicidad reduciendo los tipos de abogados a solo tres. Así, proponemos dejar de lado el poder y la influencia para atender solamente a las funciones profesionales que ejecuta cada grupo. Por razones de comodidad llamaremos genéricamente funcionarios a todos los abogados que trabajan en los organismos del Estado. Llamaremos académicos a los abogados que trabajan como profesores universitarios o como juristas profesionales (dogmáticos o teóricos) en centros de investigación y llamaremos litigantes a los abogados que se desempeñan en estudios y firmas privadas, ya sea en labores de litigación, asesoría, consejería o representación de intereses de terceros ${ }^{28}$.

En este punto es necesario hacer dos prevenciones. Por un lado, la distinción tripartita que hemos propuesto no impide que, atendiendo a objetivos de investigaciones diferentes, se puedan distinguir y analizar a otros grupos como los estudiantes de derecho, los asistentes o técnicos jurídicos o los abogados que trabajan en organismos internacionales, entre otros. Por otro lado, la división propuesta es porosa y permite la yuxtaposición de los tres grupos.

\footnotetext{
${ }^{27}$ Adler, L. \& Gil-Mendieta, J. "El neoliberalismo y los cambios en la elite de poder en México”, en Redes: Revista hispana para el análisis de redes sociales, 1, 2002, pp. 1-23.

${ }^{28}$ María Beatriz Arriagada nos ha cuestionado la necesidad de explicitar el rol de los teóricos y filósofos del derecho dentro de la comunidad jurídica. Provisionalmente sostenemos que ellos no son miembros paradigmáticos de la comunidad jurídica, en la medida en que los otros miembros del mismo grupo no siempre consideran el trabajo de los teóricos del derecho como típicamente jurídico.
} 


\subsection{La comunidad jurídica y sus textos}

La comunidad jurídica produce textos orales y escritos. Algunos de estos textos son típicos e identitarios de los abogados. Las demandas, los artículos científicos de la dogmática, los alegatos de recursos, los manuales de estudio, los contratos de compraventa de inmuebles, las objeciones en los juicios orales, son algunos ejemplos de estos textos.

Algunos de los textos de la comunidad jurídica están muy estereotipados porque la comunidad ha regulado (formal o informalmente) cómo deben elaborarse, es decir, qué partes deben componerlos y cómo debe usarse el lenguaje en cada una de esas partes ${ }^{29}$. Así por ejemplo, los miembros de la comunidad jurídica tienen expectativas preconcebidas sobre un recurso de protección: "cómo debe enfrentarse la escritura del recurso"; "cuándo es una buena oportunidad para presentar este recurso" y "cómo debe escribirse cada una de las partes del recurso", entre otras convenciones que desconocen quienes no pertenecen a la comunidad.

Usando la terminología usual en estudios del discurso usaremos la expresión género discursivo para referirnos a estos textos considerablemente regulados por la comunidad jurídica. Los géneros son "una constelación de potencialidades de convenciones discursivas, sustentadas por los conocimientos previos de los hablantes/escritores y oyentes/lectores (almacenados en la memoria de cada sujeto), a partir de constricciones y parámetros contextuales, sociales y cognitivos" ${ }^{30}$. Los géneros se expresan en eventos comunicativos (textos orales y escritos) que instancian el propósito comunicativo demandado. Un género es un type de un propósito mientras que un texto es un token, ya que todos los textos de un mismo género comparten el mismo propósito comunicativo y otros rasgos que permiten identificarlos como token de un mismo type. Los especímenes de un género son textos que han estereotipado su contenido semántico, su estructura textual y su funcionamiento pragmático. Así, su definición depende de estos tres niveles y su éxito también se juega en esos niveles. El concepto de género textual también posibilita observar la existencia de comunidades discursivas que producen, usan y consumen de forma privilegiada ciertos textos. Estas comunidades son grupos de personas que comparten un modo específico de pensar, es decir, un sistema conceptual y metodológico y una forma particular de comunicar estas nociones que son sostenidos por los miembros de la comunidad y que deben ser aprendidas por los miembros noveles ${ }^{31}$.

\footnotetext{
${ }^{29}$ Usamos el verbo usar de manera muy general y dejando entre paréntesis las controversias de filosofía del lenguaje.

${ }^{30}$ Parodi, G. Géneros académicos y géneros profesionales: Accesos discursivos para saber y hacer. Ediciones Universitarias Pontificia Universidad Católica de Valparaíso, Valparaíso, 2008, p. 26.

${ }^{31}$ Carlino, P. Escribir, leer y aprender en la universidad. Una introducción a la alfabetización académica, Fondo de Cultura Económica, México, DF., 2005, p. 92; Flower L. \& y Higgins, L. "Collaboration and the Construction of Meaning”, en Technical Report N ${ }^{\circ} 56$, National Center for the Study of Writing, University of California at Berkeley y Carnegie Mellon University, 1991.
} 
Por otro lado, los géneros están determinados por las funciones sociales que la comunidad demanda resolver a la comunidad jurídica en virtud de la división del trabajo, de modo que el rol social que ocupan los abogados y el contexto social y profesional en que ese rol se despliega imponen restricciones sobre la forma en que los individuos de cada género se estructuran, producen, consumen y circulan.

\section{LOS INTERCAMBIOS DENTRO DE LA COMUNIDAD JURÍdicA}

La comunidad jurídica se organiza alrededor de la producción de ciertos tipos de textos. Este es un segundo factor de individualización de sus miembros. Más allá de la naturaleza de su organización social y técnica, las prácticas discursivas nos permiten saber quiénes pertenecen al grupo de los abogados y a qué tipo de abogado estamos hablan$\mathrm{do}^{32}$. Así, los géneros hacen viable el intercambio comunicativo entre los miembros de la comunidad jurídica y entre ellos y quienes no son profesionales del derecho pero que integran a la comunidad normativa de referencia o de otras comunidades normativas.

Cada uno de los tres grupos de abogados que se han modelado más arriba (litigantes, académicos y funcionarios) domina géneros discursivos que les son típicos y que definen el rol y la posición relativa de ese grupo al interior de la comunidad jurídica. Los litigantes, por ejemplo, escriben demandas, recursos procesales, contratos, informes a clientes y participan en conversaciones con colegas y clientes. Por su parte, los funcionarios escriben leyes, decretos e instrucciones, dictan sentencias judiciales, reglamentos y Autos Acordados, entre otros textos. Además, los funcionarios conducen audiencias de juicio frente abogados litigantes y se comunican con los ciudadanos cada vez que resuelven una solicitud o un requerimiento. Finalmente, los académicos escriben informes en derecho, manuales, artículos científicos, libros de divulgación, comentarios de jurisprudencia y reseñas de libros. También dictan clases de pre y postgrado, asisten a congresos y dictan conferencias. Los miembros de los tres grupos pueden escribir columnas en los diarios y en sitios de noticias en Internet, conceden entrevistas y buscan influir en el debate público y político de la comunidad normativa usando los medios de comunicación social.

Siguiendo a Parodi, los géneros que cultiva la comunidad jurídica pueden ser clasificados en académicos y profesionales ${ }^{33}$. Los primeros son aquellos cuyos propósitos comunicativos preferentes son construir conocimiento científico especializado o enseñar a los miembros noveles de la comunidad cuáles son las bases del conocimiento disciplinar que sustenta la práctica profesional del derecho.

Los géneros profesionales son aquellos cuyo propósito comunicativo central es cumplir con las funciones laborales de los abogados, ya sea configurando una relación jurídica o regulando la conducta de un tercero. Naturalmente, el límite que separa lo

\footnotetext{
${ }^{32}$ Beacco, J. "Trois perspectives linguistiques sur la notion de genre discursif”, en Langages, 2004, pp. 153, pp. 109-119.

33 Parodi, G. Géneros académicos y géneros profesionales: Accesos discursivos para saber y hacer. Ediciones Universitarias Pontificia Universidad Católica de Valparaíso, Valparaíso, 2008.
} 
académico y lo profesional es difuso, ya que hay géneros que son transversales y que funcionan como vasos comunicantes entre ambas categorías. Este solapamiento impide pensar en la clasificación de los géneros como una tipología (divisiones categoriales rígidas e independientes) y exige concebir la existencia de un continuum de textos que crean, fijan y transmiten los conocimientos especializados que la comunidad sustenta, es decir, una topología ${ }^{34}$. La identificación de los géneros de la comunidad jurídica chilena es un trabajo que no se ha realizado.

Los destinatarios de los géneros son otros miembros de la comunidad normativa o de la comunidad jurídica. También es posible que se dirijan a miembros de otras comunidades normativas o comunidades jurídicas. Llamaremos legos y semilegos a los miembros de una misma comunidad normativa que, aunque no son abogados, poseen un grado variable de conocimientos sobre cuestiones jurídicas. Un trabajador social, el actuario de un tribunal, un contador, un policía y un estudiante de derecho son semilegos, mientras que un ingeniero en minas, una educadora de párvulos, un astrofísico, un periodista, un empresario y una dueña de casa son legos. Para designar genéricamente a los miembros de una comunidad jurídica, a quienes más arriba hemos agrupado como funcionarios, académicos y litigantes, usaremos la expresión expertos. Finalmente, para hablar de quienes integran una comunidad jurídica que pertenece a una comunidad normativa diferente usaremos la expresión expertos foráneos y para nombrar a quienes integran diferentes comunidades normativas pero que carecen de formación jurídica usaremos la expresión forasteros ${ }^{35}$.

Si se modelan los intercambios comunicativos dentro de una misma comunidad normativa (comunidad A) se producen ocho intercambios organizados en cuatro flujos de comunicación. Estos intercambios se diagraman en la Figura 1 representados por flechas con doble punta. Un primer flujo de comunicación se produce entre los miembros de la comunidad normativa (legos o semilegos) y los expertos. Al interior de la comunidad jurídica hay 6 intercambios diagramados como tres flujos de comunicación bidireccionales: 1) funcionario-litigante; 2) funcionario-académico y, 3) litigante-académico. A estos flujos de comunicación intergrupos se agregan otros tres flujos intragrupales.

\footnotetext{
${ }^{34}$ Parodi, G. Géneros académicos y géneros profesionales: Accesos discursivos para saber y hacer. Ediciones Universitarias Pontificia Universidad Católica de Valparaíso, Valparaíso, 2008.

${ }^{35}$ Las relaciones entre dos o más comunidades normativas y/o jurídicas implican variadas dificultades teóricas y metodológicas, aquí solo destacaremos la necesidad de modelar: i) las demandas mutuas por reconocimiento; ii) los procesos de integración, asimilación y resistencia cultural y, iii) las relaciones de influencia científica y profesional por medio de la construcción de redes sociales como las que acontecen con las migraciones.
} 


\section{Figura 1}

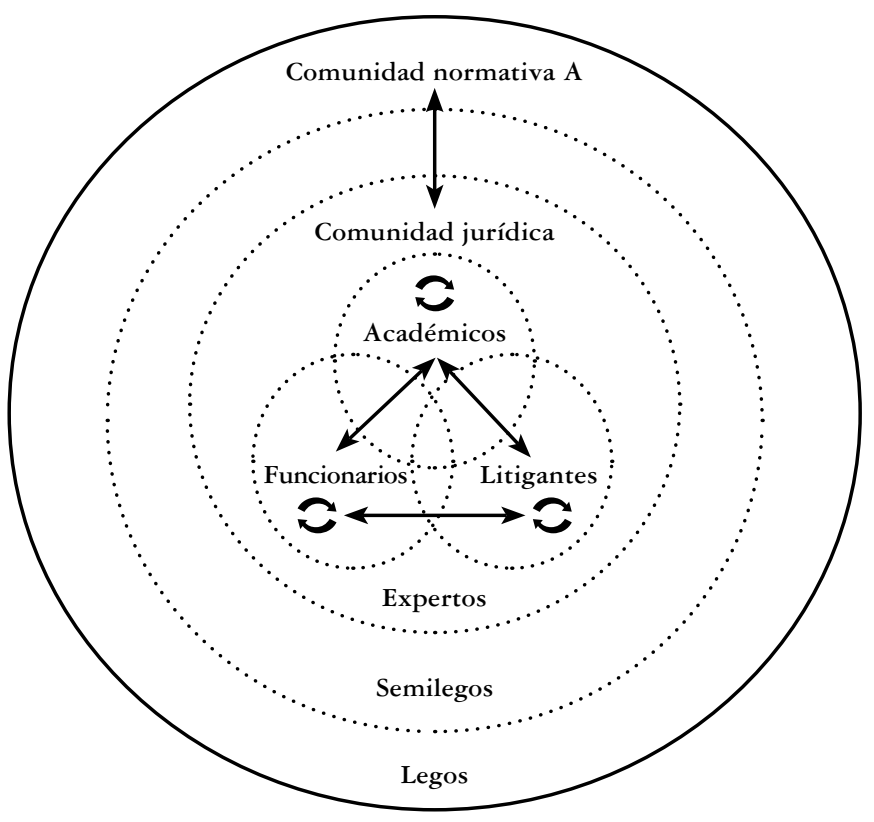

El diagrama de los flujos de comunicación (Figura 1) debe ser interpretado con seis observaciones aclaratorias.

a) Géneros discursivos cerrados. En la comunidad jurídica los géneros discursivos que configuran la identidad de los abogados son, en general, bastante cerrados, es decir, el conjunto de autores coincide cualitativa y cuantitativamente con el conjunto de receptores $^{36}$. Esto diferencia a los abogados de otras comunidades como los educadores, los periodistas, los trabajadores sociales y los profesionales de la salud, ya que en estas comunidades los géneros centrales de las disciplinas son abiertos, es decir, se dirigen a grupos de legos o semilegos. Las noticias de los periódicos; las clases ante estudiantes; las charlas a grupos de personas vulnerables y las explicaciones sobre cómo seguir un tratamiento médico son todos ejemplos de textos que se orientan a personas que no son colegas o expertos en una disciplina. En este sentido, el modelo representa dos hechos: i) los tres grupos de abogados no se relacionan del mismo modo (ni con la misma intensidad) con los legos y semilegos y, ii) el conocimiento jurídico se distribuye principalmente entre los expertos y, por ello, muchos de los géneros típicamente jurídicos se organizan de una forma muy poco accesible para las personas que no son parte de la comunidad jurídica. El grupo de los académicos es el que parece desarrollar textos

\footnotetext{
${ }^{36}$ Maingueneau, D. "Problèmes d'ethos", en Practiques No 113/114, 2002, pp. 55-67; Lotman, Y. Estructura del texto artístico. ITSMO, Madrid, 1982.
} 
casi exclusivamente orientados a satisfacer las necesidades de los otros dos grupos de abogados estando más distante de quienes no son expertos, salvo el caso de los géneros desarrollados para la enseñanza universitaria ${ }^{37}$.

b) Flujos de comunicación asimétricos. La segunda observación es sobre la capacidad del modelo para adaptarse y representar flujos prioritarios de comunicación e intercambios de bienes. Por ejemplo, si aceptamos que la dogmática es una actividad que los académicos despliegan para influir en los funcionarios (especialmente en los jueces), es forzoso considerar que el flujo de comunicación entre los académicos y los funcionarios no es simétrico. Muchos de los géneros que forman lo que se llama dogmática jurídica solo existen con el propósito de influir en los funcionarios, pero ello no ocurre a la inversa. Es claro que las decisiones de la administración del Estado o las sentencias de los jueces no se dirigen preferentemente a los académicos, sino que a los litigantes y a los semilegos y legos ${ }^{38}$. Al preguntar cómo los funcionarios responden a la presión de los académicos se aprecia la posibilidad de que los primeros administren y distribuyan bienes como el prestigio profesional entre los segundos.

c) Flujos de comunicación fuera de la comunidad normativa. La tercera observación dice relación con la capacidad de ampliar el modelo para analizar casos en donde se producen flujos de comunicación entre dos o más comunidades normativas y/o jurídicas. Por ejemplo, el análisis de los litigios internacionales entre Chile y sus países vecinos puede intentarse a partir de la diagramación del flujo de comunicación entre forasteros (los periodistas peruanos y bolivianos) y los expertos chilenos y, por otro lado, el flujo de comunicación entre los expertos chilenos y los expertos peruanos, entre otras instancias de comunicación.

d) Representación de relaciones sociales. La cuarta observación tiene que ver con la posibilidad de complejizar el modelo frente a la necesidad de representar las relaciones sociales formales e informales vinculadas a la circulación de textos. Llamamos relación social formal a un vínculo entre dos o más individuos cuya distancia social es alta y/o en donde la transacción se produce al alero de instituciones profesionales. El calificativo

\footnotetext{
${ }^{37}$ Los géneros científicos que circulan en la comunidad jurídica fijan tres problemas claves para los académicos: i) el rol de las revistas científicas y de los libros como espacios para el diálogo entre los expertos, ii) las convenciones discursivas y los valores que sustentan los estándares de rigurosidad científica y, iii) el desarrollo de la investigación en derecho y los medios por los cuales se divulgan los resultados a los no expertos.

${ }^{38}$ Esta afirmación no desconoce que la oscuridad de los géneros jurídicos para los legos es un problema de acceso a la justicia. En específico, sobre los problemas que presenta el análisis de sentencias penales chilenas hay cuatro investigaciones previas relacionadas entre sí. Agüero, C. y Zambrano, J. "Integración metodológica para el estudio del texto de las sentencias penales chilenas", en Revista Convergencia, 2010, vol. 17, No 54, pp. 69-91; Agüero, C. y Zambrano, J. "La narración en las sentencias penales”, en Revista Universum, vol. 24, No 2, 2009, pp. 28-41; Agüero, C. “¿Conforman las sentencias penales un género discursivo?”, en Estudios filológicos, N 53 [en prensa]; Agüero, C. "Una crítica al modelo de narración judicial como explicación científica”, en Ius et Praxis, vol. 20, No 1 [en prensa].
} 
informal designa entonces a aquellas relaciones en donde la distancia social entre los participantes es reducida y/o en donde la transacción no se produce en el marco de una institución profesional. Así, es posible complejizar el diagrama de la Figura 1 incluyendo relaciones familiares, de amistad, de negocios, de afinidad política o ideológica y relaciones económicas para complementar la información sobre quienes elaboran y consumen los textos jurídicos ${ }^{39}$.

La distinción entre relaciones sociales formales e informales permite observar que hay cuatro vínculos relevantes para comprender los flujos de comunicación: i) las relaciones formales e informales entre los expertos y, ii) las relaciones formales e informales entre los expertos y los no expertos. Las relaciones sociales formales entre expertos ocurren al alero de instituciones como el colegio profesional, las organizaciones gremiales o en las sociedades científicas, mientras que las relaciones informales son redes clientelares que se producen en el marco del ejercicio de la profesión. En ambos casos la relación está mediada por una tensión entre la idoneidad y el reconocimiento profesional, por un lado, y la confianza por otro.

Las relaciones formales entre expertos y no expertos acontecen en instituciones como las universidades y las organizaciones sin fines de lucro, en tanto que las relaciones informales son redes sociales vinculadas a la capacidad del individuo para ampliar y fortalecer el capital social y el cultural que le ha entregado su familia y el sistema educacional en que se fue formando (aquí también es relevante, por ejemplo, la participación en una Iglesia y en un partido político).

e) El rol de las fuentes formales del derecho. La quinta observación se refiere a considerar que las fuentes formales que regula a muchos de los géneros son una variable que modifica la representación de los flujos de comunicación dentro de las comunidades normativa y jurídica. En los géneros jurídicos profesionales que circulan entre expertos, como las demandas y las sentencias judiciales, es la legislación la que determina la existencia del intercambio comunicativo y sus objetivos principales, es decir, es el derecho el que define qué intercambios de comunicación tienen relevancia jurídica; cuáles son las condiciones que deben cumplir los intercambios para ser afortunados y producir efectos jurídicos; cuáles son los participantes obligatorios de ciertos géneros y, cuál es la forma (oral o escrita) y los modos semióticos que se usan parar crear, fijar y transmitir los contenidos.

\footnotetext{
${ }^{39}$ Las relaciones de influencia científica y profesional expresadas mediante relaciones intertextuales como las referencias y citas bibliográficas representan un ejemplo del flujo de comunicación entre académicos y funcionarios, entre académicos y litigantes y entre académicos. El análisis de estos flujos de comunicación debiese permitir responder preguntas tales como ¿por qué los académicos citan profusamente a sus colegas y tan escasamente a la investigación personal?, ¿cuáles son las preferencias discursivas que siguen los jueces al hacer referencias a obras dogmáticas?, ¿qué investigaciones académicas son las más citadas por los litigantes en sus presentaciones a los tribunales?, entre otras.
} 
f) Conocimientos previos del operador. La sexta observación se relaciona con los conocimientos que exige el uso del modelo. Quien analiza un texto jurídico específico tiene que saber qué género discursivo instancia ese texto, es decir, tiene que conocer las reglas de composición del type. Esta tarea no se puede realizar sin hacer referencia a tres dimensiones: i) cómo el texto se integra en la comunidad normativa, es decir, cómo funciona el texto con otros textos no jurídicos; ii) cómo funciona el texto en la comunidad jurídica con otros textos jurídicos y, iii) cómo se organizan las partes o elementos del texto. Así, la definición del type debe aprehender que el proceso de definición es espiral, es decir, que se inicia el sistema social, traspasa la comunidad jurídica y su vórtice se posiciona en el nivel estructural. De esta forma, las necesidades de la comunidad normativa determinan las opciones posibles dentro de la comunidad jurídica (aunque el género sea cerrado) y no a la inversa ${ }^{40}$. La Figura 2 diagrama la organización de las tres dimensiones a las que nos hemos referido.

Figura 2

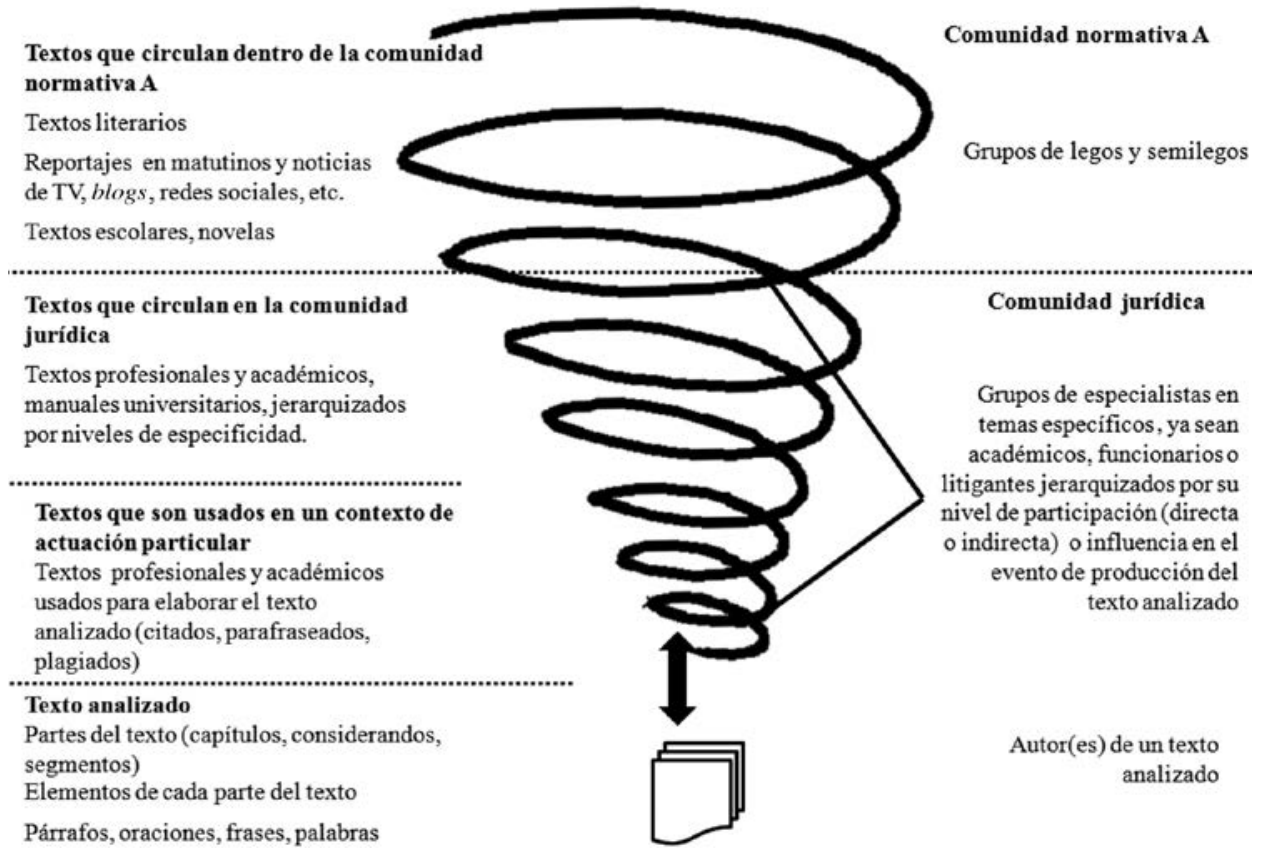

\footnotetext{
${ }^{40}$ El espiral es un diseño programático y a priori. Los datos de campo podrían corregir el modelo o forzar su abandono.
} 
La espiral propuesta permite explicar al menos cuatro tipos de preguntas diferentes: i) cómo los textos que producen los expertos en derecho se distancian y asemejan de los que circulan fuera de la comunidad jurídica pero dentro de la comunidad normativa; ii) cómo un texto singular, típicamente jurídico, se integra a la dinámica social con la mediación de otros textos elaborados por los expertos (o no); iii) cómo las relaciones entre textos reflejan las interacciones entre los miembros de las comunidades y, iv) cómo los procesos sociales de deliberación y debate al interior de una sociedad, por ejemplo, en los llamados momentos constitucionales y momentos constituyentes ${ }^{41}$, impactan en la elaboración de textos jurídicos y no jurídicos.

\section{CONCLUSIONES}

Hemos sostenido que la sociedad puede modelarse como una comunidad normativa, es decir, como un grupo de individuos en donde cada uno de los miembros es competente para realizar, grosso modo, interpretaciones morales, religiosas, políticas y jurídicas sobre los actos de otros miembros de la comunidad o de terceros que se insertan en ella. Hemos usado la expresión comunidad jurídica para designar al grupo de expertos en derecho dentro de la comunidad normativa. A su vez, dentro de la comunidad jurídica hemos distinguido tres tipos de abogados: los funcionarios, los académicos y los litigantes. Luego, mostramos que es posible modelar cómo estos tipos de abogados se comunican entre sí intercambiando bienes y servicios por medio del uso de textos.

Dentro de cada comunidad normativa el negocio de los abogados es ofrecer un intangible: la solución de los conflictos jurídicos. El servicio consiste, desde una perspectiva naïf, en usar las palabras para negociar, mediar, demandar, sentenciar, impugnar actos, celebrar actos y contratos, legislar, resolver peticiones, etcétera. Desde una perspectiva menos ingenua, no es posible desconocer que quien pronuncia las palabras usa sus redes sociales para dar fuerza al discurso que pronuncia por encargo. Así, es claro que los servicios de un abogado no solo incluyen la creación de varios tipos de textos especializados (saber qué decir, cómo decirlo, cuándo decirlo, a quién decirlo y con qué intención decirlo), sino que también la puesta en marcha de una estrategia social: la activación de un conjunto de redes (horizontales y/o verticales) que fortalecen la tecnología discursiva.

Pensar un modelo elemental para analizar la dinámica discursiva de una comunidad jurídica es interesante porque el análisis del discurso de los abogados permite mejorar nuestra capacidad de controlar el impacto de las actuaciones de este grupo de expertos sobre la comunidad normativa. Si aceptamos, tal como sostienen Petrucci y Le Goff, ${ }^{42}$ que todos los textos jurídicos son memorativos porque están cargados de las tensiones sociales imperantes en un grupo humano en un momento determinado, la construcción

\footnotetext{
${ }^{41}$ Naturalmente, estamos sobreinterpretando las expresiones de Bruce Ackerman. Ackerman, B. We the People. Foundations. Vol. 1, Harvard University Press, Cambridge, 1991.

42 Petrucci, A. La ciencia de la escritura. Primera lección de paleografía. Fondo de Cultura Económica, México DF., 2002; Le Goff, J. El orden de la memoria. El tiempo como imaginario. Paidós, Madrid, 1991.
} 
de un modelo para el análisis discursivo dentro de una comunidad jurídica puede mejorar nuestra comprensión sobre cuatro fenómenos interrelacionados: i) la contribución de los abogados al logro de ciertos ideales políticos como la Democracia y el Estado de Derecho mediante sus intervenciones en la deliberación de la sociedad civil, ii) el uso que los abogados hacen de sus influencias sociales para sustentar sus discursos académicos y profesionales, iii) las relaciones entre una determinada posición en la red social de la comunidad jurídica y la defensa de una actitud ideológica por parte de un cierto tipo de abogado y, iv) las condiciones bajo las cuales las expectativas que tienen los no expertos en derecho sobre el rol social y profesional de los abogados se ven defraudadas.

Para finalizar solo nos resta recordar una idea obvia: los textos (jurídicos y no jurídicos) cambian su morfología y sus relaciones con otros textos solo cuando ya han cambiado las necesidades comunicativas de las personas. Nunca sucede al revés. Por ello, cualquier modelo que intente disociar la relación entre los textos y la sociedad entregará siempre una respuesta parcial.

\section{BiBLIOGRAFÍA}

Ackerman, B. We the People. Foundations. Vol. 1, Harvard University Press, Cambridge, 1991.

AdLer, L. \& Gil-Mendieta, J. "El neoliberalismo y los cambios en la elite de poder en México", en Redes: Revista hispana para el análisis de redes sociales 1, 2002, pp. 1-23.

Adler, L. Lo formal y lo informal en las sociedades contemporáneas, Centro de Investigaciones Diego Barros Arana, Santiago, 2008.

AGüero, C. “¿Conforman las sentencias penales un género discursivo?”, en Estudios filológicos, $\mathrm{N}^{\circ} 53$ [en prensa].

AgüEro, C. "Una crítica al modelo de narración judicial como explicación científica", en Ius et Praxis, vol. 20, $\mathrm{N}^{\circ} 1$ [en prensa].

AGÜERo, C. y Zambrano, J. "Integración metodológica para el estudio del texto de las sentencias penales chilenas", en Revista Convergencia, 2010, vol. 17, No 54, pp. 69-91.

AgüERo, C. y Zambrano, J. "La narración en las sentencias penales", en Revista Universum, vol. 24, $\mathrm{N}^{\circ} 2,2009$, pp. 28-41.

Bailer-Jones, D. "Models, Metaphors and Analogies", en Machamer, P. \& Silberstein, M. (eds.), The Blackwell Guide to the Philosophy of Science, Blackwell Publishers, Oxford, 2002, pp. 108-127.

Barker, C. \& Galasinski, D. Cultural Studies and Discourse Analysis. A Dialogue on Language and Identity. Sage Publications, Londres, 2001.

BeAcCO, J. "Trois perspectives linguistiques sur la notion de genre discursif”, en Langages, 2004, pp. 153, 109-119.

CARLINO, P. Escribir, leer y aprender en la universidad. Una introducción a la alfabetización académica. Fondo de Cultura Económica, México, DF., 2005.

Chouliaraki, L. \& Fairclough, N. Discourse in late modernity. Rethinking critical discourse analysis. Edinburgh University Press, Edimburgo, 1999.

Conley, J.M. \& O’Barr, W.M. Rules versus relationships. The ethnography of legal discourse. The University of Chicago Press, Chicago, 1998.

De la MAZA, I. Los abogados chilenos: entre el Estado y el mercado. Juris Science master's thesis, Programa for International Legal Studies, Stanford University, publicada bajo el mismo 
título en el número 10 de la Colección de Informes de Investigación de la Universidad Diego Portales, enero de 2002. Disponible en el sitio web: http://www.udp.cl/descargas/ facultades_carreras/derecho/pdf/investigaciones/Informes_derecho_Informes_de_investigacion/10_Losabogados_Estadomercado_Inig_delaMaza.pdf [consultado el 30 de octubre de 2013].

Fletcher, G. "Law", en Smith, B. (ed.) John Searle. Cambridge University Press, Cambridge, 2003. Flower L. \& Higgins, L. "Collaboration and the Construction of Meaning", en Technical Report $\mathrm{N}^{\circ}$ 56, National Center for the Study of Writing, University of California at Berkeley y Carnegie Mellon University, 1991.

Galdia, M. Legal Linguistics. Peter Lang, Frankfurt, 2009.

GeE, J. An Introduction to Discourse Analysis: Theory and Method. Routledge, Londres, 1999.

GibBons, J. Language and the Law. Longman, Londres, 1994.

Halliday, M. \& Martin, J. Writing science. Literacy and discursive power. University of Pittsburgh Press, Pittsburgh, 1993.

Harris, M. "History and Significance of the Emic/Etic Distinction", en Annual Review of Anthropology 5, 1976, pp. 329-350.

Harris, M. "Chapter Two: The Epistemology of Cultural Materialism", en Cultural Materialism: The Struggle for a Science of Culture, Random House, New York, 1980, pp. 29-45.

Hassan, R. "Society, language and the mind: the meta-dialogism of Basil Bernsteins theory". Christie, F. (ed). Pedagogy and the shaping of consciousness. Linguistic and social processes. Continuum, Londres, 1999.

Headland, T., Pike, K. \& Harris, M. (eds). Emics and Etics: The Insider/Outsider Debate, Sage Publications, Beverly Hills, California, 1990.

Kitayama, S. \& Cohen, D. Handbook of Cultural Psychology. Guilford Press, New York, 2007.

KLEIN, G. Sources of power: How people make decisions. MIT Press, Boston, MA. 1994.

LE Goff, J. El orden de la memoria. El tiempo como imaginario, Paidós, Madrid, 1991.

LEMKe, J.L. Using language in the classroom. Oxford University Press, Oxford, 1989.

LotMAn, Y. Estructura del texto artístico. ITSMO, Madrid, 1982.

Maingueneau, D. "Problèmes d'ethos", en Pratiques No 113/114, 2002, pp. 55-67.

Matus, J. "El positivismo en el derecho penal chileno. Análisis sincrónico y diacrónico de una doctrina de principios del siglo XX que se mantiene vigente", en Revista de Derecho, vol. XX $\mathrm{N}^{\circ}$ 1, 2007, pp. 175-203. Disponible en: http://www.scielo.cl/pdf/revider/v20n1/art08.pdf

Matus, J. y Carnevalli, R. "Análisis descriptivo y cuantitativo de los artículos de Derecho penal y Criminología de autores chilenos en Revistas publicadas en Chile (1885-2006)”, en Revista Política criminal, Vol. 2, No 3, Julio, 2007, D2, pp. 1-138.

NADER, L. The life of the law. Anthropological projects. University of California Press, Berkeley, 2002.

Nelken, D. "Using the concept of legal culture", en Australian Journal of Legal Philosphy, $\mathrm{N}^{\circ} 29$, pp. 1-28. Disponible en: http://www.law.berkeley.edu/files/Nelken_-_Using_the_Concept_ of_Legal_Culture.pdf

O'TEízA, T. El discurso pedagógico de la historia: un análisis lingüistico sobre la construcción ideológica de la historia de Chile (1970-2001), Frasis Editores, Santiago, 2006.

PARODI, G. Géneros académicos y géneros profesionales: Accesos discursivos para saber y hacer. Ediciones Universitarias Pontificia Universidad Católica de Valparaíso, Valparaíso, 2008.

Petrucci, A. La ciencia de la escritura. Primera lección de paleografía. Fondo de Cultura Económica, México DF., 2002.

Pike, K. (ed.). Language in Relation to a Unified Theory of Structure of Human Behavior, Mouton, The Hague, Netherlands, 1967. 
Travers, M. \& Manzo, J.E. Law in action: Ethnomethodological and conversation analytical approaches to law. Ashgate, Aldershot, 1997.

Tocqueville, A. La democracia en América. Fondo de Cultura Económica, México DF., 2006.

Von Wright, G. Ensayos sobre explicación y comprensión. Alianza, Madrid, 1980.

Von Wright, G. Explicación y comprensión. Alianza, Madrid, 1987.

Wodak, R \& Meyer, M (Eds). Methods of Critical Discourse Analysis, Sage Publications, Londres, 2001. 